Louisiana State University

LSU Digital Commons

Faculty Publications

Department of Chemistry

$7-1-2012$

\title{
SPHERES, Jülichs high-flux neutron backscattering spectrometer at FRM II
}

Joachim Wuttke

Forschungszentrum Jülich (FZJ)

Alfred Budwig

Forschungszentrum Jülich (FZJ)

Matthias Drochner

Forschungszentrum Jülich (FZJ)

Hans Kämmerling

Forschungszentrum Jülich (FZJ)

Franz Joseph Kayser

Forschungszentrum Jülich (FZJ)

See next page for additional authors

Follow this and additional works at: https://digitalcommons.Isu.edu/chemistry_pubs

\section{Recommended Citation}

Wuttke, J., Budwig, A., Drochner, M., Kämmerling, H., Kayser, F., Kleines, H., Ossovyi, V., Pardo, L., Prager, M., Richter, D., Schneider, G., Schneider, H., \& Staringer, S. (2012). SPHERES, Jülichs high-flux neutron backscattering spectrometer at FRM II. Review of Scientific Instruments, 83 (7) https://doi.org/10.1063/ 1.4732806

This Article is brought to you for free and open access by the Department of Chemistry at LSU Digital Commons. It has been accepted for inclusion in Faculty Publications by an authorized administrator of LSU Digital Commons. For more information, please contact ir@lsu.edu. 


\section{Authors}

Joachim Wuttke, Alfred Budwig, Matthias Drochner, Hans Kämmerling, Franz Joseph Kayser, Harald Kleines, Vladimir Ossovyi, Luis Carlos Pardo, Michael Prager, Dieter Richter, Gerald J. Schneider, Harald Schneider, and Simon Staringer 


\section{AIP parterat Scientific Instruments}

SPHERES, Jülich's high-flux neutron backscattering spectrometer at FRM II

Joachim Wuttke, Alfred Budwig, Matthias Drochner, Hans Kämmerling, Franz-Joseph Kayser et al.

Citation: Rev. Sci. Instrum. 83, 075109 (2012); doi: 10.1063/1.4732806

View online: http://dx.doi.org/10.1063/1.4732806

View Table of Contents: http://rsi.aip.org/resource/1/RSINAK/v83/i7

Published by the American Institute of Physics.

\section{Additional information on Rev. Sci. Instrum.}

Journal Homepage: http://rsi.aip.org

Journal Information: http://rsi.aip.org/about/about_the_journal

Top downloads: http://rsi.aip.org/features/most_downloaded

Information for Authors: http://rsi.aip.org/authors

\section{ADVERTISEMENT}

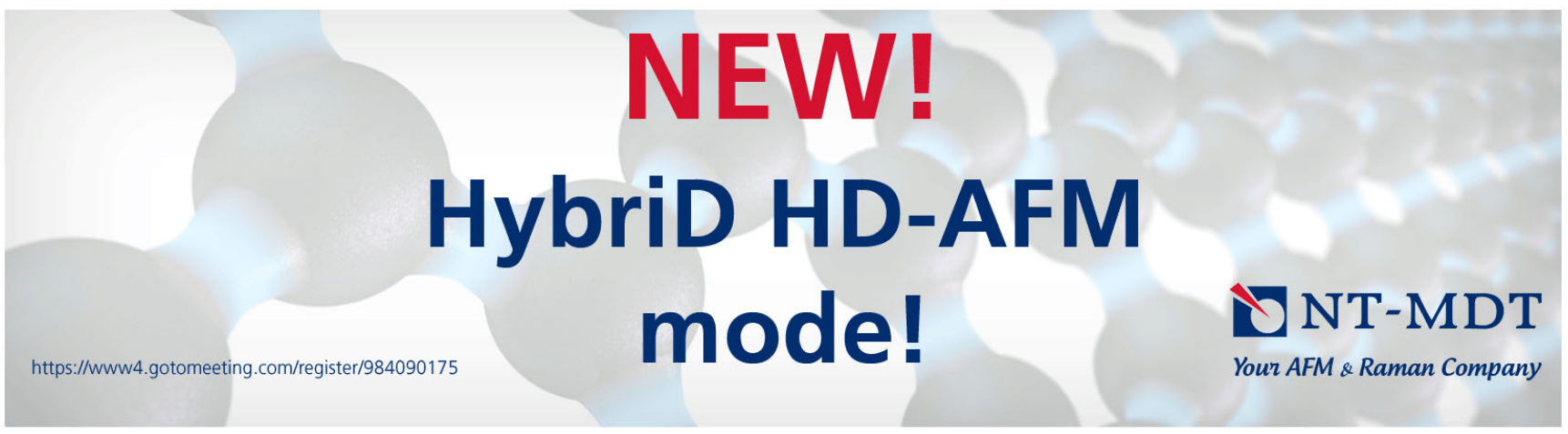




\title{
SPHERES, Jülich's high-flux neutron backscattering spectrometer at FRM II
}

\author{
Joachim Wuttke, ${ }^{\text {a) }}$ Alfred Budwig, Matthias Drochner, Hans Kämmerling, \\ Franz-Joseph Kayser, Harald Kleines, Vladimir Ossovyi, Luis Carlos Pardo, ${ }^{\text {b) }}$ \\ Michael Prager, ${ }^{c)}$ Dieter Richter, Gerald J. Schneider, Harald Schneider, \\ and Simon Staringer \\ Forschungszentrum Jülich GmbH, 52425 Jülich, Germany
}

(Received 16 April 2012; accepted 15 June 2012; published online 18 July 2012)

SPHERES is a third-generation neutron backscattering spectrometer, located at the $20 \mathrm{MW}$ German neutron source FRM II and operated by the Jülich Centre for Neutron Science. It offers an energy resolution (fwhm) better than $0.65 \mu \mathrm{eV}$, a dynamic range of $\pm 31 \mu \mathrm{eV}$, and a signal-to-noise ratio of up to 1750:1. ( 2012 American Institute of Physics. [http://dx.doi.org/10.1063/1.4732806]

\section{INTRODUCTION}

Neutron backscattering is a versatile technique for measuring the dynamics of spins, atoms, and molecules on a $\mathrm{GHz}$ scale. Typical applications include hyperfine interactions in magnetic materials, molecular rotations, diffusion, and relaxation in complex systems. ${ }^{1,2}$

Historically, three instrument generations can be distinguished. Proposed by Maier-Leibnitz in 1966 (Ref. 3), neutron backscattering was first realized in a test setup at the 4 MW reactor FRM (Garching, Germany). Count rates were of the order of $1 \mathrm{~h}^{-1}$ and the signal-to-noise ratio was 2:1 at best so that it took several weeks to measure a single spectrum. ${ }^{4}$ Following this demonstration of principle, firstgeneration backscattering spectrometers were build at Jülich (" $\pi$-Spektrometer" $)$ ) and Grenoble (IN10 (Refs. 6-8), IN13 (Ref. 9)); they were reviewed in Refs. 1 and 10-12.

In the second-generation instrument IN16 of Institut Laue-Langevin (ILL) at Grenoble, consequent use of focussing optics has been made; in it, a deflector chopper allows to operate a spherical monochromator in exact backscattering geometry. ${ }^{13-15}$ Another instrument of this type is planned to be built at the Australian research reactor OPAL (Ref. 16).

In third-generation spectrometers, the focussing optics is made even more efficient by a phase-space transform (PST) chopper. This device, invented by Schelten and Alefeld in 1984 (Ref. 17), enhances the neutron spectrum available at the monochromator, and allows for a particularly compact instrument design. The first realisation has been the highflux backscattering spectrometer (HFBS) at the National Institute of Standards and Technology (NIST, Gaithersburg, Md.). ${ }^{18,19}$

In this article, we present SPHERES, the SPectrometer for High Energy RESolution, built and operated by JCNS (Jülich Centre for Neutron Science) at FRM II

\footnotetext{
a)Electronic mail: j.wuttke@fz-juelich.de.

b) Permanent address: Grup de Caracterització de Materials, Departament de Física i Enginyeria Nuclear, Universitat Politècnica de Catalunya, Barcelona, Spain.

c) Deceased in 2008 .
}

(Forschungsneutronenquelle Heinz Maier-Leibnitz of Technische Universität München at Garching, Germany). A third PST based backscattering instrument, named IN16B, is currently under construction at the ILL where it will replace both IN10 and IN16 (Refs. 20 and 21).

The development of SPHERES has been documented in several papers. ${ }^{22-27}$ First productive experiments were performed in June 2007, and routine operation started in April 2008. Since then, two thirds of the beam time is offered to external users. Experiment proposals are invited twice a year through the JCNS user office. By now (March 2012), nearly 100 experiments have been performed, and more than $20 \mathrm{pa}-$ pers have appeared. In the following, we describe the design of SPHERES, its hardware and software components, and its performance characteristics. The supplementary material contains a list of suppliers and some technical details. ${ }^{29}$

\section{INSTRUMENT DESIGN}

\section{A. Instrument layout}

In a backscattering spectrometer, strong energy filtering of incident and scattered neutrons is achieved by Bragg reflection from perfect monochromator and analyzer crystals under angles close to $180^{\circ}$. This peculiar geometry leads to some complications in the spectrometer design; it entails the need for a primary beam deflector and a duty-cycle chopper. A particularly compact design is achieved if these two functions are realized by one and the same component: by a chopper that bears deflector crystals on its circumference. As an important side-effect, the Bragg deflection, by moving crystals, results in a PST that enhances the useable flux at the monochromator.

The resulting instrument layout is shown in Fig. 1. It is similar to the layout of the HFBS (Ref. 19), with three major differences: (1) HFBS is operated under vacuum whereas the SPHERES housing is gas tight but not vacuum proof; (2) at HFBS, the monochromator is located outside the instrument housing; (3) in the secondary spectrometer, detectors and analyzers have changed sides with respect to the primary beam, resulting in a larger scattering angle range at SPHERES. 


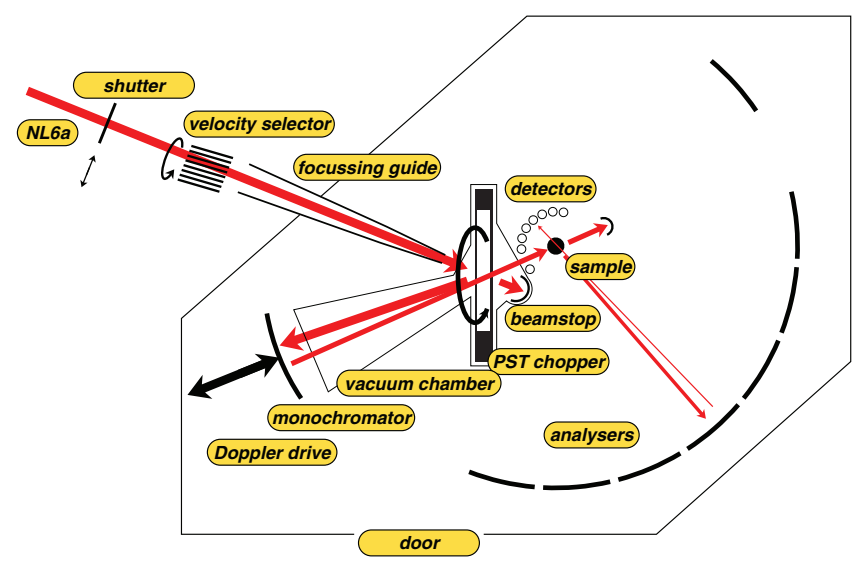

FIG. 1. Basic layout of SPHERES.

\section{B. Crystal choices}

The basic design decision for a backscattering spectrometer is the choice of the monochromator and analyzer crystals. Initially, SPHERES was foreseen to be convertible between a $\mathrm{Si}(111)$ and a $\mathrm{Si}(311)$ configuration. $\mathrm{Si}(111)$ is the standard at most backscattering spectrometers. The shorter wavelength of $\mathrm{Si}(311)$ gives access to a larger range in the scattering wave number $q$, with promising application niches in the study of diffusion on very short length scales. ${ }^{30,31}$ However, the lower resolution (which goes with $\lambda^{-2}$ ) and background problems from Bragg reflections (for instance, from sample holders or from cryostat walls) have so far prevented wide-spread usage. In commissioning SPHERES, the original mechanics had to be modified in many details in order to block neutronic background channels and to provide biological shielding. As a result, the instrument has lost its convertibility; the $\mathrm{Si}(311)$ option has been abandoned for the foreseeable future.

Another important choice is pyrolitic graphite (PG) for the deflector crystals. Once deflector, monochromator, and analzyer crystals are chosen, the neutronic parameters listed in Table I are fixed. The angle $2 \alpha_{0}$ between incoming and deflected beam is obtained from the Bragg equation $2 \cos \alpha_{0}$ $=\lambda /(c / l)$, where $c=6.710 \AA$ is the lattice constant of PG, and $l$ the order of the Bragg reflection $[00 l]$.

TABLE I. Parameters that depend on the choice of Bragg reflections for energy selection and deflection. The current configuration of SPHERES uses silicon (111) in monochromator and analyzers and pyrolitic graphite (002) as deflector.

\begin{tabular}{lcc}
\hline \hline Bragg reflection for energy selection & $\mathrm{Si}(111)$ & $\mathrm{Si}(311)$ \\
\hline Final neutron wavelength $\lambda_{\mathrm{f}}(\AA)$ & 6.271 & 3.275 \\
Final neutron energy $E_{\mathrm{f}}(\mathrm{meV})$ & 2.080 & 7.626 \\
Final neutron velocity $v_{\mathrm{f}}(\mathrm{m} / \mathrm{s})$ & 630.9 & 1208 \\
Bragg reflection for deflection & $\mathrm{PG}(002)$ & $\mathrm{PG}(004)$ \\
Deflection angle $2 \alpha_{0}\left({ }^{\circ}\right)$ & 41.65 & 25.14 \\
Schelten-Alefeld velocity $v_{0}(\mathrm{~m} / \mathrm{s})$ & 300.1 & 290.5 \\
\hline \hline
\end{tabular}
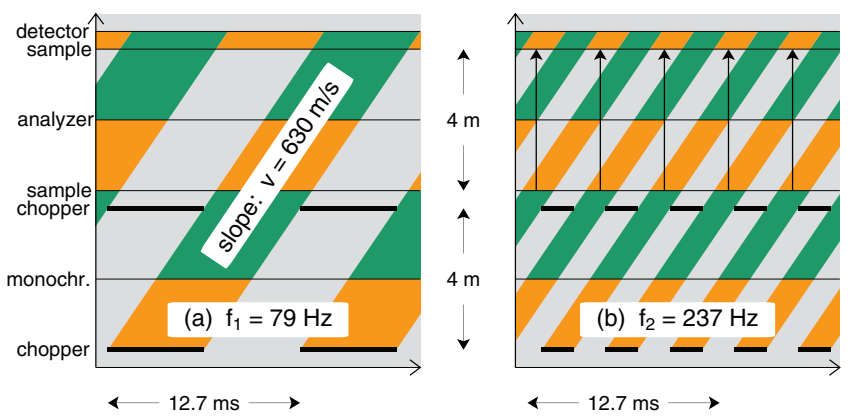

FIG. 2. Neutron path versus time-of-flight: (a) As realized in SPHERES, with chopper opening frequency $f_{1}=v_{\mathrm{f}} / 4 R$. (b) Originally planned for SPHERES (and realized in HFBS), with $f_{2}=3 f_{1}$. The upward arrows describe neutrons that are directly scattered from the sample into the detector.

\section{Time-of-flight logic}

Another decisive hardware choice is the radius $R=2000 \mathrm{~mm}$ of the analyzer spheres. This parameter fixes the time of flight $\tau:=2 R / v_{\mathrm{f}}=6.34 \mathrm{~ms}$ for a round trip from the sample to the analyzer and back. During one period $\tau$, the detectors see $n$ pulses of directly scattered neutrons, interlaced with $n-1$ pulses of energy-analyzed neutrons. If both types of pulses have the same duration $\tau_{n}$, then $\tau_{n}=\tau /(2 n-1)$, with $n=1,2, \ldots$ Accordingly, the chopper must open with a frequency $f_{n}=1 /\left(2 \tau_{n}\right)$.

In the primary spectrometer, neutrons are deflected by the closed chopper. After a round trip to the monochromator and back, they must hit the open chopper phase. Therefore, the distance chopper-monochromator $L_{\mathrm{CM}}$ must obey $2 L_{\mathrm{CM}} / v_{\mathrm{f}}=(2 m-1) \tau_{n}$. Since the monochromator should have about the same curvature as the analyzers, the only practicable choice is $L_{\mathrm{CM}}=R$ and $m=n$. All this is illustrated by the time-distance diagrams in Fig. 2.

HFBS uses $n=2$, and so will IN16B. The present chopper of SPHERES was also originally designed for $n=2$, but because of mechanical instabilities, it is only operated with $n=1$. This reduces the efficiency of the PST, but it has a positive effect upon the signal-to-noise ratio, to be explained below (Sec. VI C).

\section{INSTRUMENT COMPONENTS}

\section{A. Neutron guide and velocity selector}

SPHERES is located in the neutron guide hall West of FRM II. Like all instruments in this hall, it receives neutrons through beam tube SR1 from the cold source. SPHERES uses an end position at the secondary neutron guide NL6a. This guide has a cross section of $60 \times 120 \mathrm{~mm}^{2}$; its curved segments have a radius of $1000 \mathrm{~m}$. The guide is shared with two upstream instruments, MIRA (Ref. 28) and DNS, that use thin crystal monochromators to extract neutrons at shorter wavelengths than those needed at SPHERES.

The instrument shutter of SPHERES and its mechanical neutron velocity selector are located in two separate biological shieldings. The velocity selector (EADS Astrium) reduces the incoming neutron spectrum to about $6.27 \pm 0.34 \AA$. It is followed by a convergent neutron guide ("anti-trumpet," by 
S-DH) that reduces the beam section by a factor of 11.5 to $25 \times 25 \mathrm{~mm}^{2}$. Neutrons leave this guide system with a divergence of about $\pm 47 \mathrm{mrad}$ horizontal, $\pm 64 \mathrm{mrad}$ vertical. For details on all this, see the supplementary material. ${ }^{29}$

\section{B. Phase-space transform chopper}

The PST chopper is the key component of a thirdgeneration backscattering spectrometer. As said above, it combines the functionalities of a beam deflector and of a dutycycle chopper. Half of its circumference bears deflector crystals that redirect incoming neutrons towards the monochromator. The other half circumference is open. When the chopper is open, neutrons coming out of the neutron guide are transmitted towards a beam stop that is integrated in the chopper housing, and neutrons coming back from the monochromator are transmitted towards the sample.

To enable spectral scans, a sufficiently large wavelength band must be forwarded towards the monochromator. This requires deflector crystals with large mosaicity. PG crystals, specified for a mosaicity of $2.5^{\circ}$ fwhm (Advanced Ceramics), were selected according to rocking curves measured oneby-one on the triple-axis neutron spectrometer UNIDAS at Jülich. To further enhance the horizontal mosaicity, the crystals are assembled in stacks of three, with tilts of $0^{\circ},+2.5^{\circ}$, and $-2.5^{\circ}$ imposed by aluminum wedges (Fig. 3 ).

Intensity and width of the forwarded neutron band are further enhanced by the horizontal motion of the deflector crystals. This phase-space transformation "from white to wide" has first been suggested in a conference proceedings of limited circulation $;{ }^{17}$ an easily accessible summary has been provided in Ref. 19. The Bragg reflection, by moving mosaic crystals, compresses the energy distribution and thereby enhances the flux in the acceptance range of the backscattering monochromator. In accordance with "Liouville's theorem," the compression of the energy distribution is accompanied by a widening of the angular distribution. This is not a severe
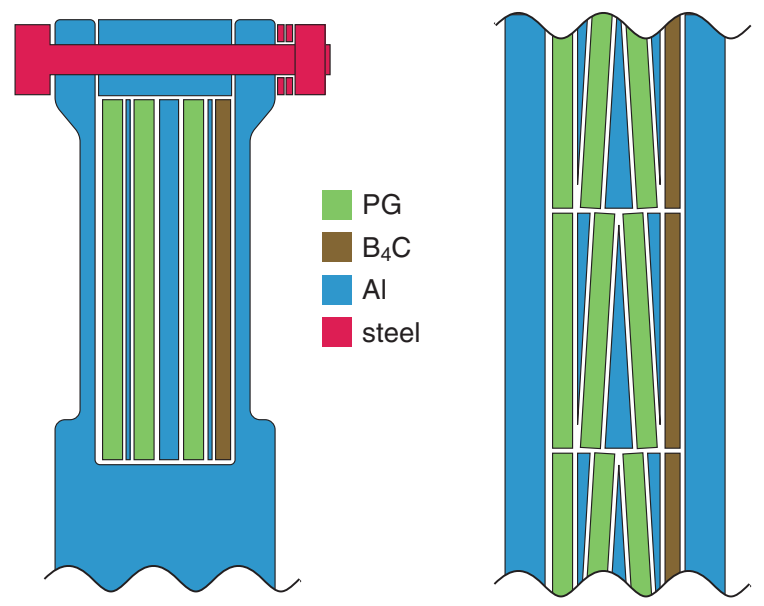

FIG. 3. Deflector crystal assembly in the PST chopper. In both drawings, the neutrons come from the left. Left drawing: cross section. Right drawing: view from outside towards the chopper axis (the closing Al element and the screws are not shown). The deflector stacks consist of three pyrolitic graphite mosaic crystals, tilted by aluminum wedges, and followed by a ${ }^{10} \mathrm{~B}_{4} \mathrm{C}$ absorber plate. (a) $79 \mathrm{~Hz}, 300 \mathrm{~m} / \mathrm{s}$

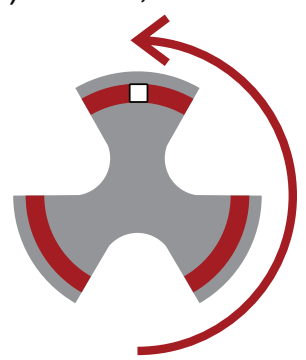

(b) $26 \mathrm{~Hz}, 100 \mathrm{~m} / \mathrm{s}$

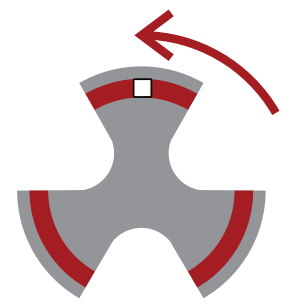

FIG. 4. Chopper operation mode, (a) originally intended, (b) currently realized. The white square shows the illuminated area. The arrows indicate the rotation during $\tau=6.34 \mathrm{~ms}$; in this time, deflected neutrons travel from the chopper to the monochromator and back.

drawback as long as the angular resolution of the entire instrument is limited by that of the secondary spectrometer.

Assuming an almost collimated incoming beam, an optimum transform is predicted for a deflector speed of

$$
v_{0}=v_{\mathrm{f}} \frac{\sin \alpha_{0}}{\cos 2 \alpha_{0}},
$$

with the neutron velocity $v_{\mathrm{f}}$ and the PG deflection angle $\alpha_{0}$ as given in Table I. For $\mathrm{Si}(111)$ and $\mathrm{PG}(002)$, one obtains $v_{0}=300.1 \mathrm{~m} / \mathrm{s}$. In the meantime, several simulations $\mathrm{s}^{19,32,33}$ and a measurement ${ }^{19}$ indicate that under realistic conditions, the optimum deflector speed is only about $250 \mathrm{~m} / \mathrm{s}$.

The current chopper rotor has a radius of $650 \mathrm{~mm}$; the crystals are located at a mean radius of $R=600 \mathrm{~mm}$. The rotor has $k=3$ crystal bearing wings of $60^{\circ}$ each. Originally, the chopper was intended to operate with a pulse fragmentation index of $n=2$ (Fig. 4(a)): Neutrons that have made the round trip chopper - monochromator - chopper would hit the second but next opening following the wing by which they had been deflected. The rotation frequency $f_{\text {rot }}=(2 n-1) f_{n} / k=f_{2}$ $=78.9 \mathrm{~Hz}$ would result in a deflector speed of $2 \pi R f_{\text {rot }}$ $=297 \mathrm{~m} / \mathrm{s}$.

Tensile forces for such $R$ and $f_{\text {rot }}$ are considerable. To keep them within admissible limits, the high-strength aluminum alloy alumold was chosen, and the geometry of the disk was optimized with finite-element calculations, resulting in a construction with three spokes and two recesses for each of the three wings. ${ }^{34}$

Thermal instabilities of the bearings and mechanical resonances prevent us from running the current rotor with the intended $78.9 \mathrm{~Hz}$. As a fallback solution, we are operating the chopper with $n=1$ and $f_{\text {rot }}=f_{1} / 3=26.3 \mathrm{~Hz}$ (Fig. 4(b)). A new rotor is currently under construction; it will have an asymmetric rotor $(k=1)$ to achieve a higher crystal speed while maintaining $n=1$. As an engineering compromise, a deflector velocity of $225 \mathrm{~m} / \mathrm{s}$ has been chosen, somewhat below the optimum, which, according to the simulations, is quite flat.

\section{Monochromator and analyzers}

In the standard configuration, the $\mathrm{Si}(111)$ reflection is used in monochromator and analyzers. Single-crystalline hexagonal wafers with a side length of $58.5 \mathrm{~mm}$ and a 
thickness of $500 \mu \mathrm{m}$ were glued onto spherical supports with a curvature radius of $R=2000 \mathrm{~mm}$. The bending stress induces a lattice-constant gradient that significantly improves the backscattered intensity for a moderate cost in resolution.

The latter can be estimated quantitatively as follows. The relative change of the lattice constant at a distance $d$ from the neutral surface is $\kappa d / R$ with a material constant $\kappa=0.776$ for $\operatorname{Si}(111) .{ }^{35}$ Assuming a rectangular distribution of lattice constants, and neglecting Darwin tails and other dynamical scattering effects, one would expect a rectangular distribution of backscattered energies with a full width of $\Delta E$ $=0.81 \mu \mathrm{eV}$. Convoluting this distribution with itself, to account for monochromator and analyzer, one obtains a triangular distribution with the same full width $\Delta E$. This is in reasonable agreement with the observed overall resolution of SPHERES, which is in first approximation a Gaussian with a full width at half maximum of about $0.62 \mu \mathrm{eV}$ (Sec. VI D).

An alternate monochromator/analyzer set uses polished wafers with a cut-in grid supposed to make them stress free. The resolution is about $0.4 \mu \mathrm{eV}$, but the flux is so much lower that this configuration is of little use, except possibly for the study of narrow tunneling or hyperfine splitting.

Additional monochromators offer the possibility of measuring with a fixed energy offset. $\mathrm{A} \mathrm{CaF}_{2}$ (111) monochromator, providing an offset of $24 \mu \mathrm{eV}$ with respect to the $\mathrm{Si}(111)$ analyzers, is already available.

The monochromator support, made of carbon-fiber reinforced plastics, has a size of $500 \times 250 \mathrm{~mm}^{2}$ and a spherical curvature of about $R+L_{\mathrm{CS}} / 2=2180 \mathrm{~mm}$, where $L_{\mathrm{CS}}$ $=360 \mathrm{~mm}$ is the distance between the chopper and the nominal sample location, so that the monochromator acts as a hollow mirror that maps the chopper's PG crystals onto the sample.

The small-angle analyzers consist of eight massive aluminum rings mounted on a common support. Each ring has a width of $95 \mathrm{~mm}$; between two rings, there is a gap of $10 \mathrm{~mm}$. The innermost ring, with an inner radius of $165 \mathrm{~mm}$, proved useless because it receives fringe intensity from the direct beam; therefore, it was covered with $\mathrm{Cd}$. The other seven rings cover scattering angles $2 \vartheta$ from $9.0 \pm 1.4^{\circ}$ to $27 \pm 1.4^{\circ}$. Typically, two rings focus into one detector.

The large-angle analyzers consist of seven shells, made of cast aluminum and finished by turning. They have a height of $2000 \mathrm{~mm}$, and a maximum width (in the scattering plane) of about $530 \mathrm{~mm}$. One shell covers a solid angle of about $60^{\circ}$ $\times 15^{\circ} \simeq 0.27 \mathrm{rad}^{2}$. The seven shells are located at scattering angles from $30^{\circ}$ to $135^{\circ}$, with only small gaps between them. There is no one-to-one correspondence between individual shells and detectors; the seven shells must rather be seen as one contiguous surface.

\section{Monochromator doppler drive}

A novel, resonance-free monochromator drive has been developed jointly by Forschungszentrum Jülich and an industrial company (Aerolas). The development costs were shared with the ILL, which received an identical drive for IN16 and for future use with IN16B. The monochromator shell is mounted on an arbor that is supported by two air bearing collars. Driven by a linear motor, it performs a sinusoidal motion. The displacement amplitude $Z$ can be chosen between \pm 25 and $\pm 75 \mathrm{~mm}$. The maximum frequency is about $F=10 \mathrm{~Hz}$, resulting in a maximum velocity amplitude of $V=2 \pi F Z=4.7 \mathrm{~m} / \mathrm{s}$.

To give an idea of the engineering difficulties, let us note that the peak acceleration is $2 \pi F V=295 \mathrm{~m} / \mathrm{s}^{2}$. The moving mass is nearly $10 \mathrm{~kg}$, and the maximum mechanical power is nearly $7 \mathrm{~kW}$. The linear motors generate a heat flux of up to $2.5 \mathrm{~kW}$ that is removed by water cooling. The peak current of each of the two motors is about $70 \mathrm{~A}$ at $600 \mathrm{~V}$; much of this electric power is recovered during deceleration.

We define the monochromator velocity $v$ to take positive values if the monochromator is moving towards the sample. In the rest frame of the monochromator, backscattered neutrons have the velocity $v_{\mathrm{f}}=630.9 \mathrm{~m} / \mathrm{s}$. In the laboratory frame, they have the energy

$$
E_{\mathrm{i}}(v)=\frac{m_{\mathrm{n}}}{2}\left(v_{\mathrm{f}}+v\right)^{2} .
$$

The analyzers select scattered neutrons with energy $E_{\mathrm{f}}=E_{\mathrm{i}}(0)$. In consequence, the sample energy gain in the scattering process is

$$
\hbar \omega=E_{\mathrm{i}}-E_{\mathrm{f}}=\left(2 \frac{v}{v_{\mathrm{f}}}+\frac{v^{2}}{v_{\mathrm{f}}^{2}}\right) E_{\mathrm{f}} .
$$

The quadratic term causes a small asymmetry in the dynamic range. With $V=4.7 \mathrm{~m} / \mathrm{s}$, accessible energy transfers $\hbar \omega$ extend from -30.9 to $31.2 \mu \mathrm{eV}$.

\section{E. Backscattering detectors}

The detector bank for large scattering angles consists of 11 counter tubes 24NH15, bought from Eurisys, filled with 4 bars of ${ }^{3} \mathrm{He}$. They have a diameter of 1 in. and a height of $210 \mathrm{~mm}$.

The tubes are mounted vertically. They are alternately arranged on two circles with radii of 110 and $130 \mathrm{~mm}$ at intervals $10^{\circ}$, so that complete coverage is achieved for an angular range of $110^{\circ}$ in the scattering plane. In front of the tubes (starting at a circle with radius $80 \mathrm{~mm}$ around the ideal sample centre), there are radial, vertical Cd collimator plates. The vertical opening of the detector block has been reduced to about $80 \mathrm{~mm}$ in order to block neutrons that do not come from the analyzers.

For small scattering angles, front-window counters 10NH3/5X P (also from Eurisys) are used, filled with 3.5 bars of ${ }^{3} \mathrm{He}$. Their diameter is 2 in., the active length is $1 \mathrm{in}$. They are mounted around the exit nose of the chopper housing.

\section{F. Diffraction detectors}

SPHERES is equipped with position-sensitive detector tubes (Reuter-Stokes) for auxiliary diffraction measurements to be performed simultaneously with the spectral scans. The tubes are located in the horizontal scattering plane in front of the analyzers; inevitably, they shield a few percent of the effective analyzer surface. At present, six tubes, with a diameter 
of $1 \mathrm{in}$. and $535 \mathrm{~mm}$ of active length, provide a coverage of scattering angles from about $30^{\circ}$ to $120^{\circ}$.

The resolution of this diffractometer is limited by the horizontal spread of incident neutron directions, which in the worst case is \pm (half monochromator width) $/ R= \pm 250 / 2000$ $= \pm 12.5 \%$. This is acceptable because we do not aim for full-fledged structure determination, but only for monitoring structural changes. ${ }^{36}$

\section{G. Instrument housing with argon filling}

The instrument housing has a surface of about $36 \mathrm{~m}^{2}$, and a volume of nearly $60 \mathrm{~m}^{3}$. Its walls and ceiling are made of steel plates of $5 \mathrm{~mm}$ thickness, anchored to a steel frame, and proven to be gas tight. Towards the inside, a $100 \mathrm{~mm}$ polyethylene layer moderates fast neutrons. The innermost cladding of walls and ceiling consists of Cd plates of $1 \mathrm{~mm}$ thickness.

The housing is reasonably gas tight and equipped with pneumatics and controls so that it can be filled with argon. This reduces losses by air scattering in the secondary spectrometer, where neutrons have to travel about $4.3 \mathrm{~m}$ through atmosphere. In the primary spectrometer, another $0.3 \mathrm{~m}$ are in atmosphere; the remaining flight path is in a vacuum chamber that prolongates the chopper casing towards the monochromator, as indicated in Fig. 1. Displacing 90\% of the air by argon leads to an intensity gain of more than $35 \%$, in good accord with expectations from tabulated neutron cross sections. For technical details of the argon filling, see the supplementary material. ${ }^{29}$

\section{H. Sample environments}

The standard sample environment, used for most experiments, is a cryostat SHI-950T (Janis). It is a closed-cycle refrigerator system with cold head and compressor manufactured by Sumitomo Heavy Industries. In the low-temperature range from $4 \mathrm{~K}$ to $320 \mathrm{~K}$, the sample is cooled via exchange gas. In the high-temperature range, from $290 \mathrm{~K}$ to $650 \mathrm{~K}$ ( $700 \mathrm{~K}$ for the limited amount of time), the system acts as a cryofurnace, with the sample in vacuum. The sample tube has a diameter of $60 \mathrm{~mm}$; samples are allowed to have diameters of up to $55 \mathrm{~mm}$.

The standard sample stick is equipped with $\mathrm{Si}$ diodes DT670A for temperatures from $4 \mathrm{~K}$ to $500 \mathrm{~K}$. A hightemperature stick with Pt 1000 thermometers covers temperatures from $10 \mathrm{~K}$ to $700 \mathrm{~K}$. Gas-loading equipment is under preparation.

Sample environments are inserted and operated from the platform on top of the instrument housing. If the tail diameter exceeds $160 \mathrm{~mm}$, the secondary spectrometer must be temporarily realigned to a sample position slightly out of the focus of the monochromator. To change the sample environment, it is unavoidable to break the argon atmosphere. Therefore, experiments with non-standard sample environments are preferentially scheduled at the beginning or at the end of a reactor cycle. So far, experiments have been performed with a dilution cryostat that gives access to temperatures down to
$30 \mathrm{mK}$. Other environments will be adapted and tried as requested by users.

\section{INSTRUMENT CONTROL AND DATA ACQUISITION}

\section{A. High-level user interface}

During regular experiments, physical access to the instrument is needed only for changing samples. All other operations can be performed remotely. A graphical user interface (GUI) allows:

- to open and close the instrument shutter,

- to start and stop the monochromator drive, and to set its velocity amplitude,

- to set the sample temperature, and to start temperature ramps,

- to start and stop the data acquisition, to save files, and to reset histograms, and

- to submit, edit, start, and stop experiment scripts.

It displays:

- warnings and error messages,

- the status of data acquisition, sample environment, and peripheral systems,

- current count rates for all detectors, separately for direct, elastic, and inelastic scattering,

- the latest acquired spectrum,

- the latest acquired diffractogram,

- the time evolution of various parameters in the last hours or days, and

- the status of experiment-script execution and the script commands ahead.

As the instrument is operated from different workplaces (cabin in the neutron guide hall, office, home), there is no simple way to maintain a traditional log book. Therefore, we have replaced paper by digital storage, using a simple wiki system $^{37}$ as our instrument log. Besides, there are automatically generated event and parameter logs.

\section{B. Instrument-control daemons}

As a leading principle in the design of our instrumentcontrol software, we requested that no neutrons should be lost because of peripheral software or network problems. Therefore, the instrument's subsystems are controlled by a number of independent background processes (daemons) that continue to work even if the connection to upper-level processes is interrupted.

The following daemons are the most important ones:

- spectral data acquisition (Sec. IV D),

- diffraction data acquisition (see the supplementary material), ${ }^{29}$

- slow control daemon (Sec. IV E),

- temperature-controller, supervising the sampleenvironment temperature controller,

- the Doppler driver, supervising the control PC supplied by the manufacturer, 
- event logger, receiving log lines from other daemons and writing them to an event $\log$, thereby documenting user interventions, experiment-script execution steps, system warnings, and error messages,

- parameter logger, querying every $30 \mathrm{~s}$ some 50 status parameters from other daemons and writing them to a parameter log,

- documentation server and raw-data postprocessor (Sec. V A),

- script engine, executing user-written experiment scripts, and

- the GUI server through which data and commands are channeled to and from the graphical user interface.

The spectral acquisition is time critical; therefore, it is written in the programming language $\mathrm{C}$, and it is running on a dedicated computer. For all other processes, computational speed is less important than the clarity of code; therefore, they are written in an expressive scripting language, Ruby.

All computers are connected by ethernet (instrument LAN). Interprocess communication (IPC) is based on TCP (transmission control protocol) messages. Each daemon is listening on a TCP port for commands given by other daemons. For instance, the spectral acquisition daemon receives commands (start, stop, save, reset) from the GUI server, and status queries from the parameter logger.

\section{Spectral acquisition electronics}

The data acquisition is done in a VME crate (Fig. 5) using components and software from Struck Innovative Systeme (SIS) (Ref. 38). The input consists of TTL pulses from the neutron-counter preamplifiers and from the optical encoders sensing the translation of the monochromator Doppler

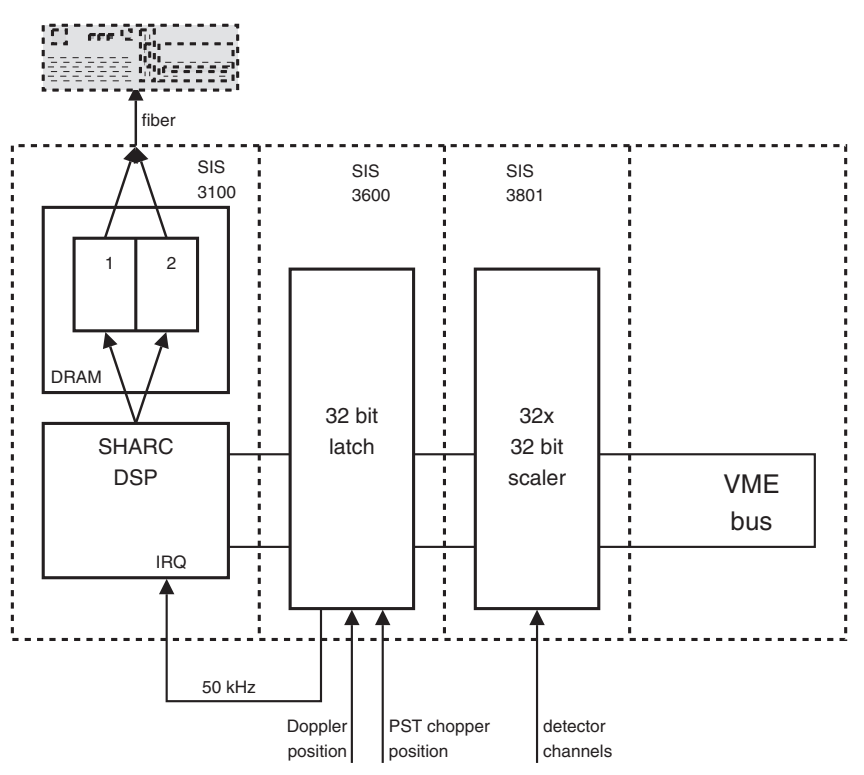

FIG. 5. The data acquisition VME crate. ${ }^{38}$ The signal processor on the SIS 3100 sequencer board is a ADSP21061. An optical fiber connects the crate to a SIS 1100 PCI card on the dedicated data-acquisition PC. drive (200 ticks per $\mathrm{mm}$ ) and the rotation of the PST chopper (2 0480 ticks per revolution). The Doppler and chopper ticks are counted up and down in home-made counter boards, then they are transmitted to a 32 bit latch. The neutron counts are transmitted via a trivial home-made interface board to a $32 \times 32$ scaler.

The sampling period is $\tau=20 \mu \mathrm{s}$. Once every $\tau$, a digital signal processor reads the data from the latch and from the scaler, writes them to DRAM, and resets the scaler. The data form a fixed-length block of twenty 32-bit words. In the following, each block shall be identified by an index $i$, corresponding to a time $t_{i}=$ const $+i \tau$. One word encodes the Doppler position $z_{i}$ and the chopper phase $\chi_{i}$ (16 bits each); the remaining 19 words $n_{j i}$ accommodate the neutron counts from up to 19 detectors $j$. The DRAM is divided into two buffers with 1000000 words each. It takes 50000 data blocks or $1 \mathrm{~s}$ to fill one buffer. While one buffer is being filled, the other buffer is read out via an optical link by the upstream PC.

\section{Spectral acquisition daemon}

The data-acquisition PC (Opteron $2 \times 2.4 \mathrm{GHz}, 64$-bit) is dedicated to running the spectral acquisition daemon. It accumulates and stores histograms containing neutron counts per energy channel and per detector. These raw spectra are only a few correction steps (Sec. V B) away from the physical scattering law $S(q, \omega)$.

The daemon consists of an endless loop that waits for one of the two DRAM buffers on the VME crate to be filled. As soon as this is the case, its contents is read via the optical link and pushed on a stack. When the stack covers at least one Doppler oscillation period, it is processed. A sine function with four free parameters (offset, amplitude, frequency, phase) is fitted to the $z_{i}$, using starting values retained from the previous fit. Then, a loop over the data blocks $i$ is performed, to be described in the next three paragraphs.

Depending on the chopper phase $\chi_{i}$, the data block is assigned a category $c\left(\chi_{i}\right)$, which can be indirect, direct, or ambiguous, depending on whether or not the neutrons have travelled the detour sample-analyzer-sample or not. In Sec. VI C, we will show how this categorization is set up. Ambiguous data blocks are not processed further.

To assign our scattering events to an energy channel $\omega$, we need to know the time

$$
\hat{t}_{i}=t_{i}-\left(v_{\mathrm{f}}+v\left(\hat{t}_{i}\right)\right)\left(L_{\mathrm{i}}^{0}-z\left(\hat{t}_{i}\right)\right)-v_{\mathrm{f}} L_{\mathrm{f}}^{c},
$$

at which the neutron has been backscattered by the monochromator. Here, $t_{i}$ is the detection time, and $L_{\mathrm{i}, \mathrm{f}}$ is the path travelled before and after scattering by the sample. The path $L_{\mathrm{f}}^{c}$ depends on the category $c$ : it is only about $10 \mathrm{~cm}$ in the direct case, but $4 \mathrm{~m}$ more in the indirect case. The path offset $z\left(\hat{t}_{i}\right)$ and the velocity modulation $v\left(\hat{t}_{i}\right)$ are given by the position and speed of the monochromator at time $\hat{t}_{i}$. This makes Eq. (4) nonlinear; it is most easily solved by iteration. For a given $\hat{t}_{i}$, the analytic derivative of the fit function $z(t)$ is used to obtain $v\left(\hat{t}_{i}\right)$. Equation (3) then yields $\omega\left(v_{i}\right)$. A quick estimate shows that the delay $\hat{t}_{i}-t_{i}$ is not 
negligible: Neutrons in the indirect category travel more than $6 \mathrm{~m}$ from the monochromator to the detector, which takes about $10 \mathrm{~ms}$, corresponding to up to $1 / 10$ of a Doppler period.

On starting the data acquisition for a given Doppler velocity amplitude, an equidistant $\omega$ mesh has been chosen, with channels $\left[\omega_{k}-\Delta \omega / 2, \omega_{k}+\Delta \omega / 2\right]$. This makes it easy to determine the pertinent channel $k(i)=\left\lfloor\left(\omega\left(v_{i}\right)-\left(\omega_{0}-\Delta \omega / 2\right)\right) / \Delta \omega\right\rfloor$. Finally, the neutron histograms of the pertinent chopper category $c\left(\chi_{i}\right)$ are incremented: $H_{j k}^{c} \leftarrow H_{j k}^{c}+n_{j i}$ for all detectors $j$. The time spent in energy channel $k(i)$ is incremented accordingly: $N_{k}^{c} \leftarrow N_{k}^{c}+1$. Here ends the loop over $i$.

All these computations must be completed before the next DRAM buffer is overwritten. After some optimizations, this requirement could be fulfilled with sufficient margin: In years of operation, not a single data block has been lost because of computer overload. As a side product, a self-contained C library for Levenberg-Marquardt leastsquares minimization has been released as an open-source project ${ }^{39}$ serving practitioners from all areas of science and engineering.

Our histogramming relies on the assumption that the motion of the monochromator accurately follows a simple analytic function. Analyses of recorded $z_{i}\left(t_{i}\right)$ traces have shown that the motion accomplished by the Aerolas Doppler drive is indeed almost perfectly sinusoidal, deviations being negligible on the scale set by the energy resolution of the spectrometer.

The accumulated histograms are written to files when a preset time interval has elapsed or when a save command is received through the daemon's IPC interface. Files are saved to the local disk, which is remote readable through the network file system (NFS). The raw-spectra, as other data produced by SPHERES, are formatted as selfdocumenting YAML files. YAML is a data-serialization specification with intrinsic support for basic data structures like arrays and hashes and with an emphasis on human readability; parsers are available for all major programming languages. ${ }^{40}$

\section{E. Safety instrumentation and slow control}

SPHERES is equipped with a fail-safe programmable logic controller (Fehlersichere speicherprogrammierbare Steuerung F-SPS, Siemens Simatic S7 300F) for safety instrumentation and slow control. ${ }^{41}$ The F-SPS is connected via an internal bus or Profibus-DP to various sensors and actors. A touchpanel next to the instrument door provides a status display; it provides the only regular user interface for safety-critical subsystems like the door lock and the argon pneumatics.

A simple daemon running on a compact-PCI computer couples the F-SPS to the instrument LAN. It has read access to all sensors and write access to safety-uncritical actors, including the shutter opener, which can be activated if the instrument housing is closed and locked and a manual switch is in "remote control" position.

\section{DATA PROCESSING}

\section{A. Raw-data postprocessor}

The raw files produced by the spectral data-acquisition daemon contain the energy mesh $\omega_{k}$, the histograms $H_{j k}^{c}$ and $N_{k}^{c}$, some information about the instrument and dataacquisition setup, and the date and time when the measurement was started and stopped. Had all other daemons crashed, this would be sufficient to allow a valid data analysis. In regular operation, however, users expect data files to contain additional information like experiment title, sample name, and sample temperature. Since the spectral acquisition daemon is deliberately kept ignorant about these things (not essential for its functioning, and potential sources of bugs and failures), the wanted information must be added expost.

This is done by the raw-data postprocessor. Running on another computer than the spectral acquisition daemon, it reads a raw-spectrum file via NFS, and looks up the start and stop time entries. Then, it opens the parameter log, reads the lines recorded during the spectral measurement, and computes mean value and standard deviation of the sample temperature and of some other parameters. Textual information like experiment title and sample name, usually entered by the user via the GUI, is retrieved from another dedicated file system. All this is written to a user-friendly aggregated raw-data file.

\section{B. Raw-data reduction with SLAW}

While some users want to work on the raw data, most ask for standard software to deliver normalized scattering laws $S(q, \omega)$. To provide this service, a new raw-data reduction program SLAW (Ref. 42) has been developed. Compared to legacy software like SQW of the ILL, SLAW offers better support for four-dimensional data sets $S(q, \omega ; T)$ or $S(q, \omega ; t)$ obtained in time-resolved inelastic scans. Also, SLAW does not bind users to a specific data format; it supports a variety of output formats, and new output routines can be easily added ad hoc.

SLAW is controlled by an input script that contains configuration commands and a map that assigns raw scans to output files. SLAW processes the raw data through the following steps of which all but the first and the last are optional:

- read raw data files, adding or subtracting subscans as needed,

- perform binning in energy, scattering angle, or time slots, possibly eliminating some of these dimensions,

- subtract empty-can measurement, optionally weighted with a self-absorption factor, ${ }^{43}$

- normalize neutron counts $H_{j k}^{c}$ to the measuring time $N_{k}^{c} \tau$ spent in a given energy channel,

- correct for incident flux variations (Sec. V C),

- divide counts by the elastic intensity of a reference measurement (usually vanadium or a low-temperature measurement with the current sample), and

- save the scattering law. 
SLAW has alternative input routines to process raw data from other backscattering spectrometers. Its structure is generic enough to allow future extension to time-of-flight spectrometers.

\section{Instead of a monitor}

Most inelastic neutron spectrometers have a hightransmission neutron counter, located a little upstream of the sample, to monitor the incoming flux. In SPHERES, the windows of such a monitor would cause undesirable background. We therefore prefer an indirect normalization.

We must account for two kinds of flux variation: temporal variations of the reactor power, of the filling of the cold source, and of the monochromator orientations of the upstream instruments, and spectral variations of the primary spectrometer transmission. The temporal variations of the incident beam can be measured with good accuracy without inserting any device into the beam: it is sufficient to monitor the $\gamma$ radiation emitted by the aluminum windows at the end of the convergent guide and at the chopper entry. Such a $\gamma$ monitor, still missing, will be installed soon.

The spectral variation of the flux at the sample is determined by a spectrum of the cold source, by the neutron guide system, by the velocity selector, and most importantly, by the PST chopper and the monochromator. To make things more complicated, the angle of the Bragg deflection in the PST chopper is energy dependent, and the monochromator is moving in space so that the geometric-optical focussing onto the sample is imperfect, with a non-trivial interplay of energy, direction, and lateral spread. To account for all this, the spectral distribution of the incident flux is best measured either by placing a neutron counter exactly at the sample position, or by counting neutrons that are scattered from the sample directly into the regular detectors. The latter method is regularly used as the "pseudomonitor" of SPHERES.

\section{PERFORMANCE}

\section{A. Flux and count rates}

The incident flux at the end of the convergent neutron guide has been measured at two occasions with gold foil activation. The results were $(1.85 \pm 0.05) \times 10^{9} \mathrm{~cm}^{-2} \mathrm{~s}^{-1}$ in November 2005, and $(1.19 \pm 0.05) \times 10^{9} \mathrm{~cm}^{-2} \mathrm{~s}^{-1}$ in February 2012. A decrease of the order of $10 \%$ was to be expected from the two breaks in the upstream neutron guide (Sec. III A). The remaining loss is not understood; it must be feared that the neutron guides have degraded substantially. To detect further degradation and to determine necessary action, it will be important to remeasure the flux more regularly in the coming years.

The flux at the sample position is more difficult to measure; for details see the supplementary material. ${ }^{29}$ The best current estimate is $\phi_{\text {sam }}=1.8 \times 10^{6} \mathrm{~s}^{-1}$ within a cross section of $25 \times 40 \mathrm{~mm}^{2}$ for the open chopper state. The average flux seen by a gold foil is only half of it.

For typical samples made for transmissions between $80 \%$ and $90 \%$, elastic count rates $\phi_{\mathrm{el}}$ are of the order $1000 \mathrm{~s}^{-1}$.
Here, as in the instrument's GUI display, such numbers are per detector, and under the assumption that all time is spent counting into this single channel. The pseudomonitor rate $\phi_{\text {dir }}$ of neutrons that are directly scattered from the sample into the detector, is about twice as large. Reasons why $\phi_{\mathrm{el}} / \phi_{\mathrm{dir}}$ is smaller than 1 include: solid angle mismatch (the detector block has a larger solid angle than the analyzers, to ensure that all backscattered neutrons have a chance to reach a detector), absorption and scattering losses in the sample (which is traversed a second time by backscattered neutrons), losses along the $4 \mathrm{~m}$ from the detector to the analyzers and back, imperfect energy selection in the monochromator, and finite backscattering probability in the analyzers.

\section{B. Dark counts}

Dark count rates in each of the large-angle detectors are of the order of $0.02 \mathrm{~s}^{-1}$ when the reactor is off, and $0.1 \mathrm{~s}^{-1}$ when the reactor is at $20 \mathrm{MW}$ and the instrument shutter closed. This is negligible compared to the background produced in our primary spectrometer.

When the instrument shutter is open and the chopper is running, but the exit window of the chopper (just before the sample position) is closed by a Cd mask, then the dark count rates in the large-angle detectors are about $0.53 \mathrm{~s}^{-1}$. Only a small part of this background is due to slow neutrons that penetrate from the primary into the secondary spectrometer, circumventing the chopper exit window. Most of the background is independent of the chopper phase, which indicates an origin between the shutter and the chopper. This has been confirmed by experimental tests: probably, a $n-\gamma$ conversion in the supermirror coating of the focussing neutron guide is followed by a conversion to fast neutrons in the guide's lead shielding.

\section{Chopper modulation of count rates}

Along with the duty-cycle chopper, a backscattering spectrometer needs a time-of-flight logic to discriminate neutrons that are backscattered by the analyzers from neutrons that are scattered directly from the sample into the detectors. In SPHERES, this time-of-flight discrimination is performed by the spectral acquisition daemon (Sec. IV D), based on the chopper phase reading that is saved every $20 \mu \mathrm{s}$ along with the Doppler position reading and the neutron counts.

The parametrization of the discrimination must be determined empirically. A chopper-phase histogram is saved in each raw data file. This histogram is based on a rectangular grid with 120 chopper-phase channels, but only two energy channels, to distinguish roughly between elastic and inelastic scattering. Figure 6 shows such data from a resolution measurement. There is a range of nearly $60^{\circ}$ with very high count rates, independent of the Doppler velocity, due to direct scattering. There is another range, labelled indirect, where almost all neutrons have been backscattered by an analyzer, as can be inferred from the strong difference between the elastic and the inelastic channel. And there are 


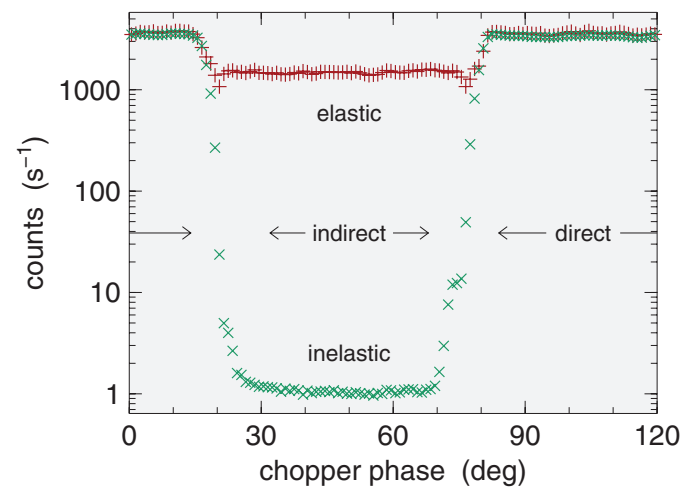

FIG. 6. Neutron counts versus chopper phase. Data from the same lowtemperature resolution measurement as in Fig. 7. In the direct phase, neutrons are scattered without energy analysis from the sample into the detectors. In the indirect phase, registered neutrons come from the analyzer. If the scattering is purely elastic, as in this example, then the inelastic signal is just undesired background.

intermediate, ambiguous ranges where directly scattered neutrons form an acceptable background in the inelastic channels. Part of this transitory range is due to the finite time it takes the chopper to open and to close. Other contributions include the following:

- In the present chopper, the graphite crystals are kept in place by aluminum corner pieces. When these pieces cross the primary neutron beam, they cause incoherent scattering into $4 \pi$, thereby sending a diffuse neutron cloud into the secondary spectrometer.

- Some directly scattered neutrons are retarded by phonon downscattering, causing a sample-dependent extension of the chopper-closing phase.

- Multiple scattering (first scattering in the sample, second scattering by the sample environment or somewhere in the secondary spectrometer) also presents a sample-dependent contribution to the extended chopper-closing phase.

To parametrize the data acquisition daemon, the instrument responsible must make a relatively arbitrary choice as to which range of chopper phases shall be considered as indirect. In general, the users of SPHERES are more interested in an excellent signal-to-noise ration than in maximum count rates. Therefore, a relatively strict chopper-phase discrimination that favors signal-to-noise ratio on the expense of total count rates is preferred. For most measurements in the past years, we have set the indirect interval to $35^{\circ}$, which means that the duty cycle (the fraction of time actually used for incrementing scattering histograms) is no more than $35 / 120=$ 0.29 .

For the future, one might consider a small modification of the data acquisition code to simultaneously record energy histograms for different chopper-phase discrimination settings. Users could then decide ex post whether to analyze a "strict" data set with optimum signal-to-noise ratio, or a "loose" data set with higher count rates.

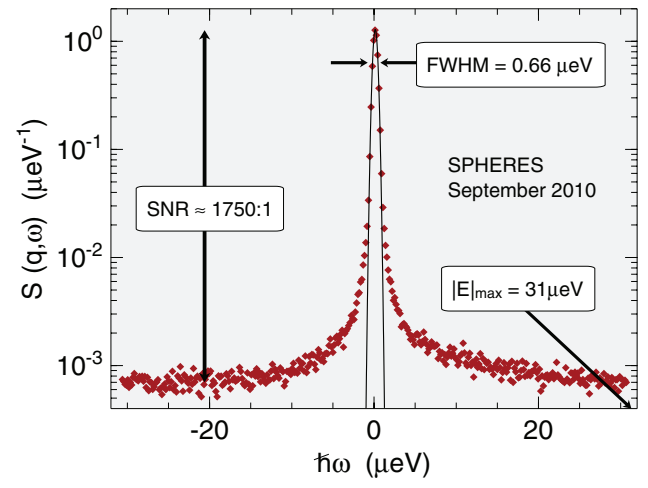

FIG. 7. A resolution measurement with a user-provided sample (a powder of hydrogen-rich organic crystals at $3 \mathrm{~K}$, in a flat cell calculated for $90 \%$ transmission). Cumulated data from the six large-angle detectors with the best signal-to-noise ratio. The solid line is a Gaussian fit to the central region $|\hbar \omega|<0.6 \mu \mathrm{eV}$.

\section{Resolution and signal-to-noise ratio}

The instrumental resolution can be empirically determined by measuring the elastic scattering of a solid. The scattering should be predominantly incoherent to guarantee sufficient intensity at all scattering angles. Vanadium, the elastic incoherent reference scattering par excellence, is not well suited because its absorption cross section at $\lambda=6.27 \AA$ is more than three times larger than its scattering cross section. Therefore, we prefer a simple plastic foil (Hostaphan RN 250: a polyethylene terephthalate foil of $0.25 \mathrm{~mm}$ thickness) as our reference scatterer.

In most experiments, however, the resolution is measured on the user sample, cooled to a low temperature where almost all scattering is elastic. This has the advantage that the sample geometry is exactly the same as in the production measurements.

Figure 7 shows such a resolution spectrum from a user experiment. The Doppler drive was running with the maximum velocity amplitude of $4.7 \mathrm{~m} / \mathrm{s}$, resulting in a dynamic range of $\pm 31 \mu \mathrm{eV}$. To determine the resolution width and the elastic amplitude, the centre of the elastic peak $(|\hbar \omega|$ $<0.6 \mu \mathrm{eV}$ ) has been fitted by a Gaussian, which is an excellent approximation though there are pronounced nonGaussian wings at larger energy transfers. The fwhm is obtained by multiplying the standard deviation of the Gaussian with $\sqrt{8 \ln (2)}$.

When the Doppler drive is run with small to moderate velocity amplitudes, the resolution at large scattering angles is typically between 0.62 and $0.65 \mu \mathrm{eV}$. At the maximum Doppler frequency used here, the resolution is slightly reduced (to $0.66 \mu \mathrm{eV}$ ), probably because of distortions of the monochromator support.

The signal-to-noise ratio (SNR) depends obviously on how we define the "noise." If we average only over energies far off the central peak, say $|\hbar \omega| \geq 20 \mu \mathrm{eV}$, then we obtain in the present example 1750:1. The SNR also depends on the sample: if the sample is too thin, then the sample-independent constant background contributes relatively more; if the sample is too thick, then many backscattered neutrons are lost when they retraverse the sample. 
TABLE II. Comparison of the three backscattering spectrometers IN16 of the ILL, ${ }^{13-15,44}$ HFBS at NIST, ${ }^{19}$ and SPHERES of the JCNS at FRM II.

\begin{tabular}{lccc}
\hline \hline Instrument & IN16 $^{\mathrm{a}}$ & HFBS & SPHERES \\
\hline Reactor power $(\mathrm{MW})$ & 58 & 20 & 20 \\
PG $(002)$ speed $(\mathrm{m} / \mathrm{s})$ & 75 & 250 & 100 \\
Si $(111)$ thickness $(\mu \mathrm{m})$ & 700 & 750 & 500 \\
Resolution fwhm $(\mu \mathrm{eV})$ & $0.8-0.9$ & 0.93 & $0.62-0.65$ \\
Flux at sample $^{\mathrm{b}}\left(10^{6} \mathrm{~s}^{-1}\right)$ & 1.2 & 2.4 & 1.8 \\
Rescaled flux $^{\mathrm{c}}$ & 0.17 & 0.91 & 1 \\
Signal-to-noise ratio $^{\mathrm{d}}$ & $1000^{\mathrm{e}}$ & 600 & 1750 \\
\hline
\end{tabular}

aith the unpolished, deformed monochromator/analyzer set that is used in almost all experiments.

${ }^{\mathrm{b}}$ While chopper open. Au foil activation gives half of this value.

${ }^{c}$ Flux at sample, divided by resolution width and reactor power, arbitrarily normalized to the value of SPHERES.

${ }^{\mathrm{d}}$ Best value reported for a non-absorbing sample of optimum thickness.

${ }^{\mathrm{e}}$ Not mentioned in the instrument descriptions, ${ }^{13-15,44}$ but confirmed by instrument users.

\section{COMPARATIVE STATUS AND PERSPECTIVE}

In Table II, some design and performance parameters of the three backscattering spectrometers IN16, HFBS, and SPHERES are compared. These parameters are intercorrelated as follows.

The instrumental resolution is within 5\% proportional to the chosen thickness of the $\mathrm{Si}(111)$ monochromator/analyzer wafers. This confirms the fundamental importance of the crystal choice for the instrument performance, and it shows that all three instruments very closely attain the optimum resolution allowed by their crystal sets.

Ceteris paribus, the flux at the sample should be proportional to the reactor power. In a very good approximation, it should also be proportional to the width of the energy band selected by the monochromator. Therefore, the figure of merit that describes the overall efficiency of the cold source, of the neutron guides, and of the primary spectrometer is flux divided by reactor power divided by resolution width. The comparison of this rescaled flux shows how much the compact design of HFBS and SPHERES, due to Schelten and Alefeld, ${ }^{17}$ is superior to the double-deflector layout of IN16.

The primary spectrometer transmission is also somewhat related to the signal-to-noise ratio: to maximize the latter, we installed in SPHERES ad hoc several slits that cut away some $10 \%$ of the incoming and of the backscattered beam in order to prevent neutrons from being transmitted through the closed chopper. The next chopper, currently under construction, will hopefully allow us to take away these slits and to increase the flux at the sample accordingly. Furthermore, in this new chopper, the PG(002) deflector crystals will be moved with about optimum speed, which will result in yet another important gain in flux.

At small scattering angles, analyzers cannot be aligned to exact backscattering geometry because detectors must be placed outside the incoming neutron beam. This makes the resolution much wider and more assymetric than in the regular backscattering detectors. Supported by simulations, we have improved the resolution at small angles by breaking the azimuthal symmetry of the analyzer rings, as will be described separately. ${ }^{45}$

\section{ACKNOWLEDGMENTS}

Building and commissioning SPHERES has been funded by the German Bundesministerium für Bildung und Forschung (Project Nos. 05NX8CJ1 and 03RI16JU1). Foundations for SPHERES were laid by former project scientists Oliver Kirstein and Peter Rottländer, and by former project engineer Tadeusz Kozielewski. Many other colleagues from the Forschungszentrum Jülich contributed to this project. We would like to acknowledge Ulrich Probst, Helga Straatmann, and Thomas Koppitz for the development of the chopper rotor, Ulrich Giesen and Ulrich Pabst for a vibrational analysis thereof, Christoph Tiemann for his contribution to the Doppler drive, Gerd Schaffrath for the instrument housing, Manfred Bednarek for the electrical installations, Peter Stronciwilk and Marco Gödel for mechanical constructions, $\mathrm{Vu}$ Thanh Nguyen for the accurate gluing of the silicon crystals, Harald Kusche, Andreas Nebel, and Björn Poschen for technical support, and Alexander Ioffe for coordinating the JCNS outstation at FRM II. Sergej Manoshin and Alexander Ioffe contributed a simulation of the convergent neutron guide. Michaela Zamponi, the new instrument responsible since fall 2011, contributed to the last Au activation measurement.

We are grateful to Winfried Petry, Jürgen Neuhaus, and all the staff of FRM II for their hospitality. We thank Harald Türck for generous help at all levels of engineering, Christian Breunig for the neutron guide, Philipp Jüttner for the shutter construction, Helmut Zeising, Birgit Wierczinski, and their entire team for the smooth handling of the radioprotection necessities, and Ralf Lorenz for sensibly supervising our safety. We thank our colleagues and friendly concurrents Bernhard Frick, Tilo Seydel, and Lambert van Eijck of the ILL for stimulating discussions and the open exchange of experiences and ideas. Victoria García Sakai, Goran Gasparovic, and Dan A. Neumann kindly showed us HFBS at NIST, and Andreas Meyer provided insights from his commissioning experience.

${ }^{1}$ M. Bée, Quasielastic Neutron Scattering (Adam Hilger, Bristol, 1988).

${ }^{2}$ R. Hempelmann, Quasielastic Neutron Scattering and Solid State Diffusion (Clarendon, Oxford, 2000).

${ }^{3}$ H. Maier-Leibnitz, Nukleonik 8, 61 (1966).

${ }^{4}$ B. Alefeld, M. Birr, and A. Heidemann, Naturwiss. 56, 410 (1969).

${ }^{5}$ B. Alefeld, Kerntechnik 14, 15 (1972).

${ }^{6}$ G. Allen, R. E. Ghosh, A. Heidemann, J. Higgins, and W. S. Howells, Chem. Phys. Lett. 27, 308 (1974).

${ }^{7}$ J. C. Cook, W. Petry, A. Heidemann, and J. F. Barthélemy, Nucl. Instrum. Methods Phys. Res. A 312, 553 (1992).

${ }^{8}$ O. G. Randl, H. Franz, T. Gerstendörfer, W. Petry, G. Vogl, and A. Magerl, Physica B 234-236, 1064 (1997).

${ }^{9}$ A. Heidemann, I. Anderson, M. Prager, and W. Press, Z. Phys. B 51, 319 (1983).

${ }^{10}$ T. Springer, Philos. Trans. R. Soc. London, Ser. B 290, 673 (1980).

${ }^{11}$ B. Alefeld, T. Springer, and A. Heidemann, Nucl. Sci. Eng. 110, 84 (1992).

${ }^{12}$ A. J. Dianoux, Physica B 182, 389 (1992).

${ }^{13}$ B. Frick, A. Magerl, Y. Blanc, and R. Rebesco, Physica B 234-236, 1177 (1997).

${ }^{14}$ B. Frick and M. Gonzalez, Physica B 301, 8 (2001).

${ }^{15}$ B. Frick, Neutron News 13, 15 (2002).

${ }^{16} \mathrm{~N}$. de Souza et al., Emu project, see http://www.ansto.gov. $\mathrm{au} /$ research/bragg_institute/facilities/instruments/emu_-_high-resolution backscattering_spectrometer. 
${ }^{17}$ J. Schelten and B. Alefeld, in Proceedings of the Workshop on Neutron Scattering Instrumentation for the SNQ, Maria Laach, 3-5 September 1984, edited by R. Scherm and H. Stiller (Berichte der Kernforschungsanlage Jülich, Jül-1954) (KFA, Jülich, 1984).

${ }^{18}$ P. M. Gehring and D. A. Neumann, Physica B 241-243, 64 (1998).

${ }^{19}$ A. Meyer, R. M. Dimeo, P. M. Gehring, and D. A. Neumann, Rev. Sci. Instrum. 74, 2759 (2003).

${ }^{20}$ M. A. Gonzalez and B. Frick, Appl. Phys. A 74s1, 474 (2002).

${ }^{21}$ B. Frick, H. N. Bordallo, T. Seydel, J.-F. Barthélémy, M. Thomas, D. Bazzoli, and H. Schober, Physica B 385-386, 1101 (2006).

${ }^{22}$ O. Kirstein, M. Prager, H. Grimm, and D. Richter, J. Neutron Res. 8, 119 (1999).

${ }^{23}$ O. Kirstein, M. Prager, T. Kozielewski, and D. Richter, Physica B 283, 361 (2000).

${ }^{24}$ O. Kirstein, T. Kozielewski, M. Prager, and D. Richter, Physica B 291, 310 (2000).

${ }^{25}$ O. Kirstein, T. Kozielewski, and M. Prager, in Proceedings of the Deutsche Neutronenstreutagung 2001, edited by T. Brückel (Materie und Material, Vol. 8), FZ Jülich, Jülich (2001).

${ }^{26}$ O. Kirstein, T. Kozielewski, M. Prager, and D. Richter, Appl. Phys. A 74, S133 (2002).

${ }^{27}$ P. Rottländer, T. Kozielewski, M. Prager, and D. Richter, Physica B 350, e823 (2004).

${ }^{28}$ SPHERES shares the guide NL6a with the monochromator of MIRA 2 (3-6 A). The alternate setup MIRA 1 uses an end position at the heavily curved guide NL6b (radius $84 \mathrm{~m}$ ) to obtain very cold neutrons (8-30 A).

${ }^{29}$ See supplementary material at http://dx.doi.org/10.1063/1.4732806 for more details on instrument components (suppliers, neutron guides, argon filling), on the diffraction data acquisition, and on the estimation of flux and count rates.

${ }^{30}$ A. Meyer, J. Wuttke, W. Petry, A. Peker, R. Bormann, G. Coddens, L. Kranich, O. G. Randl, and H. Schober, Phys. Rev. B 53, 12107 (1996).
${ }^{31}$ A. V. Skripov, J. C. Cook, D. S. Sibirtsev, C. Karmonik, and R. Hempelmann, J. Phys. Condens. Matter 10, 1787 (1998).

${ }^{32}$ M. Hennig, B. Frick, and T. Seydel, J. Appl. Crystallogr. 44, 467 (2011).

${ }^{33}$ J. Wuttke, "Theory of Bragg reflection by moving crystals and optimisation of a phase-space transform chopper," Rev. Sci. Instrum. (to be published).

${ }^{34}$ U. Probst, H. Kämmerling, T. Kozielewski, H. Straatmann, P. Rottländer, and T. Koppitz, European patent (EP) application 1706876 (2005).

${ }^{35}$ A. D. Stoica and M. Popovici, J. Appl. Crystallogr. 22, 448 (1989).

${ }^{36}$ J. Combet, B. Frick, O. Losserand, M. Gamnon, and B. Gurard, Physica B 283, 380 (2000).

${ }^{37}$ A. Gohr et al., Dokuwiki, see http://www.dokuwiki.org/ (2004-2011).

${ }^{38}$ M. Drochner, H. Kleines, M. Kirsch, T. Kozielewski, M. Prager, and P. Rottländer, in Proceedings of the 14th IEE-NPSS Real Time Conference, Stockholm, 2005.

${ }^{39} \mathrm{~J}$. Wuttke, Imfit - a $\mathrm{C} / \mathrm{C}++$ routine for Levenberg-Marquardt minimization with wrapper for least-squares curve fitting, based on work by B. S. Garbow, K. E. Hillstrom, J. J. Moré, and S. Moshier, see http://joachimwuttke.de/lmfit.

${ }^{40}$ O. Ben-Kiki, C. Evans, and I. döt Net, see http://www.yaml.org for "YAML ain't markup language."

${ }^{41}$ H. Kleines, F. Suxdorf, M. Drochner, P. Rottländer, M. Prager, T. Kozielewski, and A. Budwig, in Proceedings of the KEK, 2005-18, WEP25 (2005).

${ }^{42}$ J. Wuttke, SLAW: Neutron scattering histograms to scattering law converter, http://apps.jens.fz-juelich.de/slaw.

${ }^{43} \mathrm{~J}$. Wuttke, absco: absorption corrections for $\mathrm{x}$-ray and neutron diffraction, http://apps.jcns.fz-juelich.de/absco.

${ }^{44}$ See http://www.ill.eu/instruments-support/instruments-groups/instruments/ in16/characteristics/ for IN16 characteristics.

${ }^{45} \mathrm{~J}$. Wuttke, "Optimization of Small-Angle Geometry in the Neutron Backscattering Spectrometer SPHERES," Rev. Sci. Instrum. (to be published). 